\title{
Smart System for Human Presence Detection and Alerting Gas Leakage
}

\author{
Akshay M. Chaudhari \\ Student of BE Computer \\ Engineering, BVCOE \& RI, \\ Nashik, India, University of \\ Pune
}

\author{
Tufel K. Sayyad \\ Student of BE Computer \\ Engineering, BVCOE \& RI, \\ Nashik, India, University of \\ Pune
}

\author{
Swapnil A. Gawali \\ Student of BE Computer \\ Engineering, BVCOE \& RI, \\ Nashik, India, University of \\ Pune
}

\author{
Abhishek P. Sonar \\ Student of BE Computer \\ Engineering, BVCOE \& RI \\ Nashik, India, University of \\ Pune
}

\author{
Kavita S. Kumavat \\ Assistant Professor, BVCOR \& RI \\ Nashik, India, University of \\ Pune
}

\begin{abstract}
IoT is a spreading system of physical gadgets that are connected with various sorts of sensors and with the assistance of availability to the web, they can trade information. Through IoT, web has now stretched out its underlying foundations to practically every conceivable thing present around us and is not any more constrained to our PCs and cell phones. Security, the basic worry of any venture, has not been left untouched by IoT. This paper depicts the development of a framework that utilizations data from a few home mechanization gadgets, to distinguish the nearness of a man in the space where the gadgets are found. The recognition however doesn't depend on the data of gadgets that expressly recognize human nearness, similar to movement finders. Building up a model that continually screens the gas spill with the assistance of the electronic sensors. This information is set aside a few minutes through constant encourages over the web.
\end{abstract}

\section{Keywords}

IoT, Arduino UNO, PIR sensor, MQ2 Sensor, Prediction.

\section{INTRODUCTION}

Internet of Things point towards making life less complex via mechanizing each little errand around us. As much is IoT helping in mechanizing errands, the advantages of IoT can likewise be reached out for upgrading the current wellbeing measures. Wellbeing has dependably been an essential criterion while planning home, structures, ventures and additionally urban areas. The expanded grouping of certain gasses in the air can turn out to be amazingly perilous. Internet of Things point towards making life less mind boggling by means of automating every little errand around us. As much is IoT helping in automating errands, the points of interest of IoT can similarly be connected for redesigning the present prosperity measures. Prosperity has constantly been a basic criterion while arranging home, structures, wanders and moreover urban regions. The extended gathering of certain gasses in the air can end up being incredibly hazardous. Home automation is also come under IoT. Its various application makes lifestyle comfortable and easier way such as to reduce the power consumption and making home secure. To do work effectively and timely manner. To help the physically challenged peoples. It is helpful for the security purpose. Scope of this project can be expanded to many areas by not restricting to only home it can also be used in office, hospitals.

1.1 Arduino UNO: The Arduino UNO is microcontroller board base on the ATmega328 (datasheet). It has 14 digital input/output pins (of which six can be use as PWM outputs), six analog input, a $16 \mathrm{MHz}$ ceramic resonator, a USB connection, power jack, an ICSP header, and a reset button. It contains every think needed to support the microcontroller; simply connect it to a computer with a USB cable or power it with an AC -to- DC adaptor or battery to get started. A microcontroller board that comes pre-programmed with a boot loader that allows to upload new code to it in Arduino Software IDE without the use of an external hardware programmer. The PIR sensor and gas sensor is connected to this board for processing the sensor readings according to the program. Arduino sends the sensor data to server. The Piezo Buzzer used as an audible alarm and a $5 \mathrm{~V}$ relay used for the power cut off feature are also mounted on this board. The Arduino UNO board shown in fig. 1

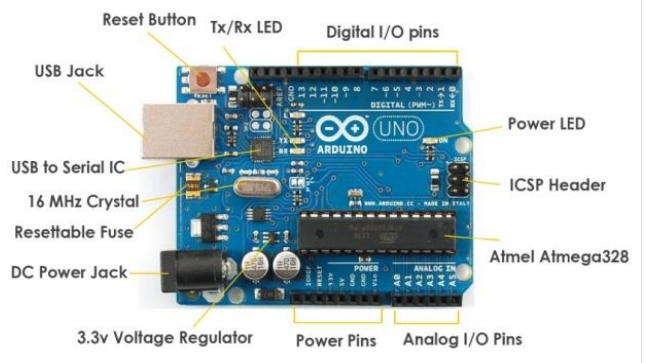

Fig 1: Arduino UNO

1.2 Gas Sensor-MQ2: The model of gas identifier system in figure 2 uses a MQ2 sensor of the MQ-x family that perceives LPG (Liquefied Petroleum Gas) noticeable all around. The sensor esteem just mirrors the approximated pattern of gas fixation in an allowable blunder go, it represents the correct gas focus. The identification of specific segments noticeable all around generally requires a more 
exact and expensive instrument. The fixation detecting scope of $300 \mathrm{ppm}$ to $10,000 \mathrm{ppm}$ is appropriate for spill location. The sensor can work at temperatures from -10 to $50^{\circ} \mathrm{C}$ and devours under $150 \mathrm{~mA}$ at $5 \mathrm{~V}$.

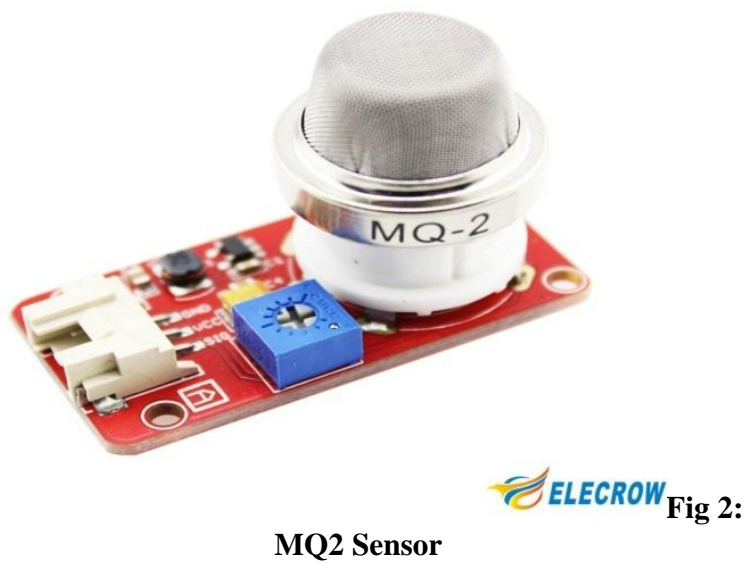

1.3 Piezo Buzzer: Piezo buzzer is used for generating audio alarm. Figure 3 shows Piezo buzzer. It firstly connected to Arduino UNO microcontroller. Working of Piezo Buzzer: MQ2 sensor sensing the gas (LPG gas) then send the value to Arduino UNO then Arduino UNO circuit machine same value then send the signal to Piezo Buzzer. Incoming the signal of Piezo Buzzer the output the sound of Buzzer.

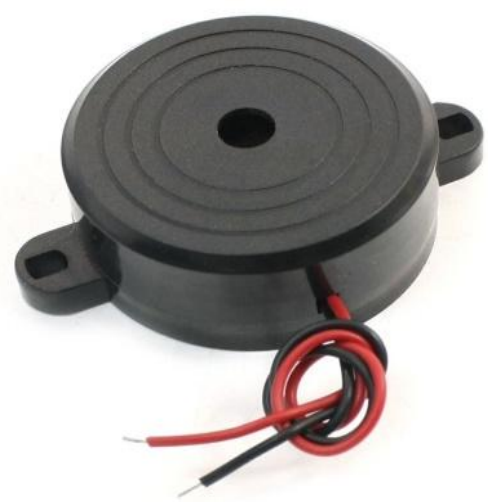

Fig 3: Piezo Buzzer

1.4 PIR sensor: A passive infrared sensor (PIR sensor) is an electronic sensor that measures infrared (IR) light emanating from objects in its field of view. They are frequently utilized as a part of PIR-based movement indicators. All term detached in this occurrence alludes to the way that PIR gadgets don't produce or transmit any vitality for identification purposes. They work completely by distinguishing the vitality radiate by other question. PIR sensors don't distinguish or measure "warm"; rather they identify the infrared radiation transmitted or reflected from a question. The PIR sensor range is Up to 10 meters at an angle of \pm 15 degree. It is shown in fig. 4 .

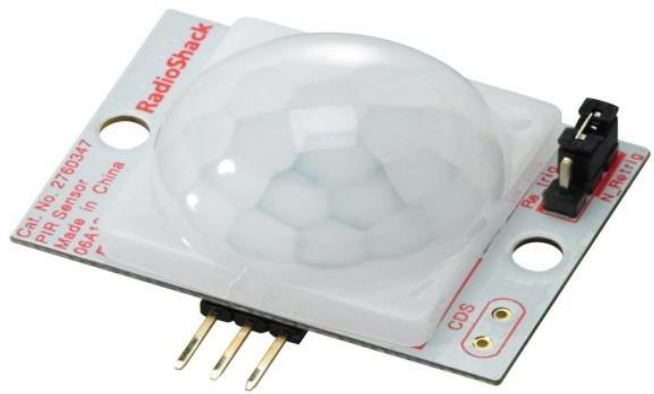

Fig 4: PIR Sensor

\section{RELATED WORK}

A conventional gas leakage system uses on-site alarms as a warning to indicate the leakage. The leakage can ignite and cause explosion. Therefore, the leakage detection of gases has gained more interest in recent years especially in fields of safety, industry, environment, and emission control. Gas leakage detectors built with microcontrollers to detect and send information through wireless media is presented [1] - [5]. The detection of certain components in the air usually requires a more precise and costly instrument, which cannot be done with a single gas sensor [7]. Steel Mesh filters out the suspended particles so that only gaseous elements are able to pass to insides of the sensor [8]. Target of this undertaking is to build up a movement sensor alert in light of a Passive InfraRed (PIR) sensor. Expect to manufacture a sensor framework which is transmit and get the flag. The movement recognition utilizing Infra-Red sensor in remotely. Other than that, it likewise went about as an auto control exchanging framework. At the point when the sensor is set off, the flag will transmit remotely to make additionally move. It will identify with auto control ON lights. At the point when the sensor detects a human movement in the sensor's identification zone, sensor will be activated and after that the room's light will automatically switch ON. Light will automatically OFF when the client was going out from the room. For whatever length of time that Passive Infra-Red sensor does not recognize movement in the identification territory, light are not work. Once the sensor is activated, framework will have around 2 minutes to run the capacity. Following 2 minutes and sensor does not recognize any movement, light will be turned OFF automatically [9]. Bhopal gas tragedy was an example of leakage accident in India. The world's 1st worst gas leakage industrial accident. On the night of December 2, a modern industrial carelessness in Bhopal prompted an arrival of 30 tons of dangerous methyl isocyanate (MIC) gas, exposing nearly about 5 lakh individuals in the city to the fatal gas. A state government report said the mishap caused more than 3500 deaths. Furthermore, more than 8,000 died till now. These were not by any means the only causalities of the accident [12]. 


\section{SYSTEM ARCHITECTURE}

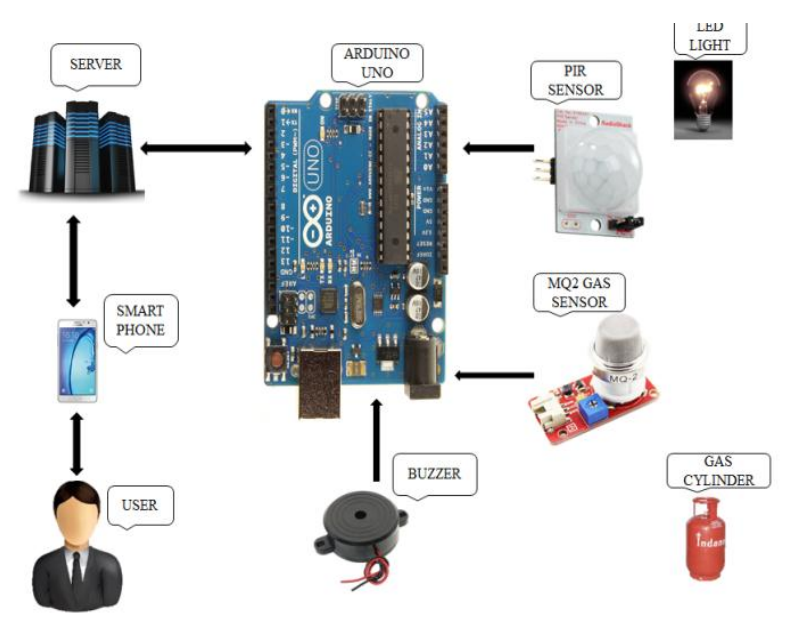

Fig 5: System Architecture

Figure 5 shows system architecture in which user smart phone is connected to the server using internet. Arduino UNO board provide control to PIR sensor and MQ2 gas sensor.

If PIR Sensor detect Human Body in room then it sends signal to Arduino UNO result of glow light of Room. Else human body not detect the PIR Sensor send signal Arduino UNO room light glow.

If MQ2 gas sensor detect the LPG gas (LPG GAS Leakage) it will send signal to Arduino UNO and Drive the alarming system connected with Buzzer. Bearing in mind user accessibility and convenience, the system made to produce alarm while buzzers represent audible alarm meant to draw immediate attention of user also it send a warning message to user on mobile phone and other one to fire brigade through server which is connected to Arduino UNO board.

\section{ALGORITHM}

\subsection{AES Algorithm: AES calculation are extraordinarily utilized for security based.}

It supersedes the Data Encryption Standard (DES), which was distributed in 1977. The calculation depicted by AES is a symmetric-key calculation, which means a similar key is utilized for both scrambling and decoding the information. In the United States, AES was reported by the NIST as U.S. FIPS PUB 197 (FIPS 197) on November 26, 2001.

\subsection{AES calculation function:}

How AES encryption functions. ... Each figure scrambles and decodes information in pieces of 128 bits utilizing cryptographic keys of 128-, 192-and 256-bits, separately. The Rijndael figure was intended to acknowledge extra square sizes and key lengths, however for AES, those capacities were not embraced.

\subsection{AES secure:}

The figure AES-256 is utilized among different places in SSL/TLS over the Internet. It's considered among the best figures. In principle, it's not crackable since the blends of keys are enormous. Despite the fact that NSA has ordered this in Suite B, they have likewise suggested utilizing higher than 128-piece keys for encryption.

\subsection{AES algorithm steps:}

1. Derive the set of round keys from the cipher key.

2. Initialize the state array with the block data (plain text).

3. Add the first round key to the starting state array.

4. Perform nine round of state manipulation

5. Perform the tenth and last round of state manipulation.

6. Copy the final state array out as the final data (cipher text).

7. For decryption reverse the above steps.

\section{CONCLUSION}

Hence this system provides human presence detection and alerting gas leakage. Human presence detection is done by using PIR sensors and gas leakage detection is done by using MQ2 sensor. The attempt while making this to make smart light control not for just saving energy but also for making smart home which are very point today in smart city projects. The gas leakage detector is promising in the field of safety. The attempt while making this prototype has been to bring a revolution in the field of safety against the leakage of harmful and toxic gases to minimize and hence nullify any major or minor hazard being caused due to them. Liquefied petroleum gas is a flammable gas, which has the potential to create a hazard. Allows the automation of several action such as turn on device when someone arrives, turn off when they leave. Allows learning the user's routines in a non-intrusive way, since it is fundamental to know when a user is at home or when he is simply interacting with the device remotely. Gas leakage detection and smart alerting and prediction based IoT using MQ2 sensor. The system can be further improved but still it performs well in its current state and shows a promising capability for detection using unspecific data from several IOT devices.

\section{REFERENCES}

[1] J. YeomJ. Yeom et al., "Enhanced toxic gas detection using a MEMS preconcentrator coated with the metal organic framework absorber," Micro Electro Mechanical Systems,. IEEE 21st International Conference on, Tucson, AZ, 2008, pp. 232-235

[2] P. Krishnan and D. Jackson, "Multifunctional gas detection based temperature compensation and data fusion," International Conference on Control, Instrumentation, Communication and Computational Technologies, Kumaracoil, 2015, pp. 796-803.

[3] Z. Yu, "Design of Combustible Gas Detectors in Industrial Premises," Intelligent Systems Design and Engineering Applications (ISDEA), Fifth International Conference on, Hunan, 2014, pp. 352-355.

[4] W. Chung, and D. Lee, "Real time multi-channel gas leak monitoring system using CPLD chip," Sensors and Actuators B, Vol. 77, 2001. pp. 186-189.

[5] V. Diduck, "Integrated local or remote control liquid gas leak detection and shut-off system," United States patent No. 6025788, 2000.

[6] L. Fraiwan, K. Lweesy, A. Bani-Salma and N. Mani, "A wireless home safety gas leakage detection system," 1st 
Middle East Conference on Biomedical Engineering, Sharjah, 2011, pp. 11-14.

[7] Grove-Gas Sensor (MQ) on seeed website. [Online]. Available: http://wiki.seeed.cc/Grove- Gas_Sensor-MQ2, 2016

[8] "How Gas Sensors Work on Engineers garage". [Online]. Available: http://www.engineersgarage.com/insight/how-gassensorworks, 2016.

[9] "MOTION DETECTION USING PIR SENSOR", IJSDR, Volume 1, Issue 5, May 2016

[10] "Gas Leakage Detection and Smart Alerting and Prediction Using IoT Second International Conference On Computing and Communications Technologies"(ICCCT'17)

[11] "A Study of Vision based Human Motion Recognition and Analysis" International Journal of Ambient Computing and Intelligence Volume 7, Issue 2, JulyDecember 2016.

[12] "Bhopal gas tragedy on $2^{\text {nd }}$ Dec 1984" http://indiatoday.intoday.in/gallery/bhopal-gas-tragedy30th-anniversary-warren-anderson/1/13577.html.

\section{AUTHOR'S PROFILE}

Akshay M. Chaudhari he is Engineering student of Computer Engineering at Brahma Valley College of Engineering And Research Institute, Nasik under University of Pune. His interest in the field of security.

Tufel K. Sayyad he is Engineering student of Computer Engineering at Brahma Valley College of Engineering and Research Institute, Nasik under University of Pune. His interest in the field of security.

Swapnil A. Gawali he is Engineering student of Computer Engineering at Brahma Valley College of Engineering and Research Institute, Nasik under University of Pune. His interest in the field of security.

Abhishek P. Sonar he is Engineering student of Computer Engineering at Brahma Valley College of Engineering And Research Institute, Nasik under University of Pune. His interest in the field of security.

K. S. Kumavat, Ph.D. pursuing under Mumbai University, ME, BE Computer Engg. Was educated at Pune University. Presently she is working as a Assistant Professor in Computer Department of Brahma Valley College of Engineering and Research Institute, Nasik, Maharashtra, India. She has presented papers at National and International conferences and also published papers in National and International Journals on various aspects of Computer Engineering and Networks. Her areas of interest include Computer Networks, Security, IoT and Advance Database. 